as that observed in nutrient broth ${ }^{2}$, showing an exponential decrease, as described by equation (1), down to $10^{-3}$ or $10^{-4}$ survivors, followed by a bend.

The effects described have been shown for coliphages $12 r^{+}, 13$ and $14 r$. Further experiments on the inactivation mechanism are now in progress.

State Serum Institute, N. K. JERNE

Copenhagen. Aug. 20. ' Hershey, A. D., Kalmanson, G., and Bronfenbrenner, J., J. Immunol.,

2 Delbrück, M., J. Bact., 50, 2, 137 (1945).

\section{Presence of Trypanosomes in Bush Babies after Eating Infected Rats}

Two bush babies (Galago crassicaudatus lasiotis Peters), examined six weeks after capture, were found heavily infected with polymorphic trypanosomes. Inquiries elicited that a few weeks earlier they had escaped from their cages at night and eaten the bodies of several white rats killed while infected with a virulent strain of $T$. gambiense and left lying on a bench. As an experiment, another bush baby and a white rat heavily infected with $T$. rhodesiense were placed in the same cage. The rat was immediately seized, killed and eaten by the bush baby, which afterwards became infected. When tested the trypanosomes failed to penetrate mucous membranes, so were presumably transmitted through abrasions; the infections were fatal within a month.

Bush babies are known to be actively carnivorous, and zur Strassen ${ }^{1}$ has stated they are very formidable enemies of small animals. Although it is not known whether bush babies can be a reservoir of trypanosomes in Nature, recent work in East Africa implicates them in the jungle yellow fever cycle; it is conceivable the infection could be acquired if rodents or other small animals harbouring the virus were eaten during dry periods when transmission by mosquitoes is interrupted.

I have to thank the Director of Medical Services, Kenya, for permission to publish this communication.

Division of Insect-Borne Diseases, R. B. HeIsCH

Medical Research Laboratory, Kenya.

Aug. 20.

${ }^{1}$ zur Strassen, O., "Brehm's Tierleben", Leipzig, 4 th ed., 13 (1925).

\section{Recombination in Bact. coli $K$ 12: Uni-} directional Transfer of Genetic Material

THE development of nutritionally independent prototroph colonies from mixed cultures of doubly dependent mutant strains of Bact. coli $K 12$ was first demonstrated in $1946^{1}$. Back mutation to prototrophism did not occur when the mutants were cultured separately. Since the pattern of unselected marker characters in prototrophs was usually different from that in either mutant, the phenomenon was clearly due to genetic recombination. The incompetence in recombination of culture filtrates (unlike type transformation in Pneumococcus), and recent evidence for the occasional occurrence of diploid heterozygous prototrophs ${ }^{2}$, strongly support the current theory that the genetic transfer is mediated by sexual conjugation. Attempts to reproduce the phenomenon in other strains and species have failed, though successful out-crossing of $K 12$ mutants with a strain of Bact. acidi lactici has been reported ${ }^{3}$.

In the following experiments, $K 12$ mutants 58-161, requiring biotin and methionine, and $W 677$, requiring leucine, threonine and aneurin, were employed. An attempt was made to investigate the dynamics of recombination by adding streptomycin at intervals to a series of plates of basal medium (plus aneurin) seeded with a mixture of 58-161 and a streptomycin-resistant mutant of $W 677\left(W 677 / S^{r}\right)$. It was anticipated that the streptomycin would rapidly block the recombination mechanism by inactivating 58-161, while allowing resistant prototroph cells formed prior to its addition to develop into colonies. In practice, the number of prototroph colonies did not differ greatly whether streptomycin was incorporated in the basal medium before plating or was added up to four hours later. Since similar results were obtained when the mutants were mixed for the first time during plating, the occurrence of recombination in mixtures before contact with streptomycin was excluded. Either prototrophs arose before the action of streptomycin on the sensitive mutant became effective, or else those functions of the cell affected by streptomycin were not involved in recombination.

Logarithmic-phase broth cultures of 58-161 wer $\theta$ treated with either 1,000 or $2,000 \mu \mathrm{gm} . / \mathrm{ml}$. streptomycin for periods up to $18 \mathrm{hr}$., under conditions optimal for bactericidal effect. Washed saline concentrates of treated cultures $(58-161 / S t)$, although frequently sterile, invariably stimulated prototroph formation when mixed with $W 677 / S^{r}$ on basal medium containing $200 \mu \mathrm{gm} . / \mathrm{ml}$. streptomycin. Whenever streptomycin treatment failed to produce sterility, control reconstruction experiments showed that at least a thousand times as many untreated 58-161 cells as those which had survived treatment were necessary for prototroph formation under similar conditions.

Mixtures of streptomycin-treated $W 677$ ( W 677/St) and $58-161 / S^{r}$, on the other hand, invariably failed to produce prototrophs, although comparable recombination-rates were given by the mixtures $\left(W 677 / S^{r}+\right.$ 58-161) and (58-161/ $\left.S^{r}+W 677\right)$. In these experiments streptomycin was not incorporated in the basal medium, since previous analysis of proved prototrophs had shown that about 95 per cent carried the $S^{r}$ or $S^{s}$ character of $W 677$. The clear-cut distinction between $58-161 / S t$ and $W 677 / S t$ in ability to participate in recombination was shown to be independent both of the presence of the $S^{r}$ character in the complementary mutant and of the basal medium environment. Thus (58-161/St $+W 677)$ produced prototroph colonies on every occasion, whether cultured directly on basal medium or initially on nutrient agar. The mixture $(W 677 / S t+58-161)$, however, failed to do so repeatedly on basal medium and, in a single experiment, when seeded on nutrient agar.

It is unlikely that sensitive cells which have been acted upon for $18 \mathrm{hr}$. by very high concentrations of streptomycin can still participate in cytoplasmic fusion in the continued presence of the drug. Moreover, if conjugation under these conditions was possible for 58-161/St, it might also be assumed for $W 677 / S^{t}$. Yet suspensions of the latter are inactive in recombination. It is more probable that recom. bination is mediated by genetic elements, extruded by the viable cell, which adhere to the cell wall and which, like viruses, are unaffected by streptomycin. 